\title{
A Cellular Automata Model for Urban Traffic with Multiple Roundabouts
}

\author{
Younes Regragui ${ }^{\mathrm{a}}$, Najem Moussa ${ }^{\mathrm{a}, *}$ \\ ${ }^{a}$ LAROSERI, Department of Computer Science, Chouaib Doukkali University, El Jadida, Morocco.
}

\begin{abstract}
Urban transportation with multiple roundabouts is facing significant challenges such as traffic congestion, gridlock and traffic accidents. In order to understand these behaviors, we propose a two-dimensional cellular automata (CA) model, where all streets are two-way, with one lane in each direction. To allow the turning movement, a roundabout is designed for each intersection where four roads meet. The distance between each pair of roundabouts is configured with the parameter $K$ while the turning behavior of drivers is modeled by a parameter $\gamma$. To study the impact of these different parameters on the urban traffic, several traffic metrics are considered such as traffic flow, average velocity, accident probability and waiting time at the entrance of roundabout. Our simulation results show that the urban traffic is in free flow state when the vehicle's density is low enough. However, when the density exceeds a critical density $\rho_{c}$, the urban traffic will be in gridlock state whenever $\gamma$ is nonzero. In the case where $\gamma=0$, the urban traffic presents a phase transition between free flow and congested state. Furthermore, detailed analysis of the traffic metrics shows that the model parameters $(\gamma, K)$ have a significant effects on urban traffic dynamics.
\end{abstract}

Keywords: cellular automaton, traffic flow, city, roundabout, gridlock.

\section{Introduction}

Traffic congestion has attracted the attention of many scientists because they are becoming one of the biggest problems found in urban environments. In order to understand traffic congestion phenomenon, scientists have carried out many studies based on different models and methods. The cellular automaton (CA) model developed by Nagel and Schreckenberg (NaSch) [1] is one of efficient models for traffic flow problems.

Traffic flow is governed by several factors, among them drivers' behavior (e.g., imperfect driving styles, slower cars and car accidents) which play an important role in the formation of traffic jams within the transportation system, especially when it is combined with the increase in car density (for a review, see $[2,3])$. Indeed, when the density of cars increases, a phase transition from free flow phase to congestion phase occurs. In free flow phase, cars move close to the speed limit, and an increase in density leads to an increase in traffic flow. However, the congested phase is characterized by a negative linear relationship between traffic flow and density. It has been shown that, in congested phase, the drivers' behavior has a major effect on the spatial distribution of vehicles relative to each other over the road $[4,5]$.

\footnotetext{
*Corresponding author: N. Moussa

Email addresses: regragui.y@ucd.ac.ma (Younes Regragui), moussa.n@ucd.ac.ma (Najem Moussa)
} 
The modern roundabout is a type of intersection with no traffic lights. It is a circular intersection with design features that offer benefits in terms of circulation safety, delays and capacity. It was first developed in the United Kingdom and now is widely used in most countries. Drivers approaching a roundabout must reduce their speeds and look for potential conflicts with vehicles already in the circle. Many technical reports stated that the average accident risk at roundabouts have found important reductions in crashes compared to other conventional crossroads or T-junction, with or without traffic signals [6-8]. However, car accidents inside roundabouts are one of the most frequent contributing factors to jam formation. Among the reasons for car accidents is the high velocity and the violation of some safety conditions related to priority rules. Despite the demonstrated safety benefits of roundabouts, some crashes still occur. An Institute study of crashes at 38 roundabouts in Maryland found that collisions occurred more frequently at entrances to roundabouts than within the circulatory roadway or at exits [9]. The researchers concluded that unsafe speeds were an important crash factor, since drivers may not have seen the roundabout in time to slow down sufficiently.

Several models have been proposed to study the characteristics of vehicular traffic flow at a roundabout. The authors in [10] have investigated the characteristics of traffic at an isolated roundabout in the framework of cellular automata and car-following models. They show that overall delay is significantly affected by roundabout size. In [11] the authors proposes Multi-stream Minimum Acceptable Space (MMAS) Cellular Automata models to study unsignalised multi-lane (two- or three-lane) urban roundabouts. They found that traffic flow is influenced by the topology of the roundabout, turning rates and critical arrival rates. On the other hand, in [12], the author proposed a cellular automaton model to analyse traffic flow around a traffic roundabout. Based on the observation of different transitions, including the transition between free-flowing and gridlock and bottleneck and gridlock, the author has identified that the gridlock can be taken as an extreme limit of bottleneck.

Cellular automata models have been also used to study the probability of car accidents for various traffic scenarios [13-15]. The first CA model including dangerous situations (accident) have been proposed by Boccara et al [13]. In [14], the author introduces the conditions for the occurrence of car accidents, based on the delayed reaction time of the successor car. The author discusses the effect of these conditions on the probability of traffic accidents. In [15], the authors have studied the probability of entering/circulating car accidents to occur at single-lane roundabout when the incoming vehicles ignore the priority rules.

The urban traffic, where traffic lights are installed to regulate traffic flow at intersections, has been well studied. Here we can cite some basic references which show that the traffic undergoes a phase transition when the density of cars in the city is increased $[16,17]$. To the best of our knowledge, no study has provided a model for urban traffic with roundabouts. In this paper, we introduce a two-dimensional CA model based on discrete events capable of simulating the traffic in urban with roundabouts. Fig. 1 gives an illustration of our proposed structure of urban environment. Our objective is to provide a study of the impact of turning movement and some geometric factor on urban traffic capacity with multiple roundabouts.

The rest of the paper is organized in the following way. We first describe our proposed model in section 2. In Section 3, we present the simulation results and the paper is concluded in Section 4. 


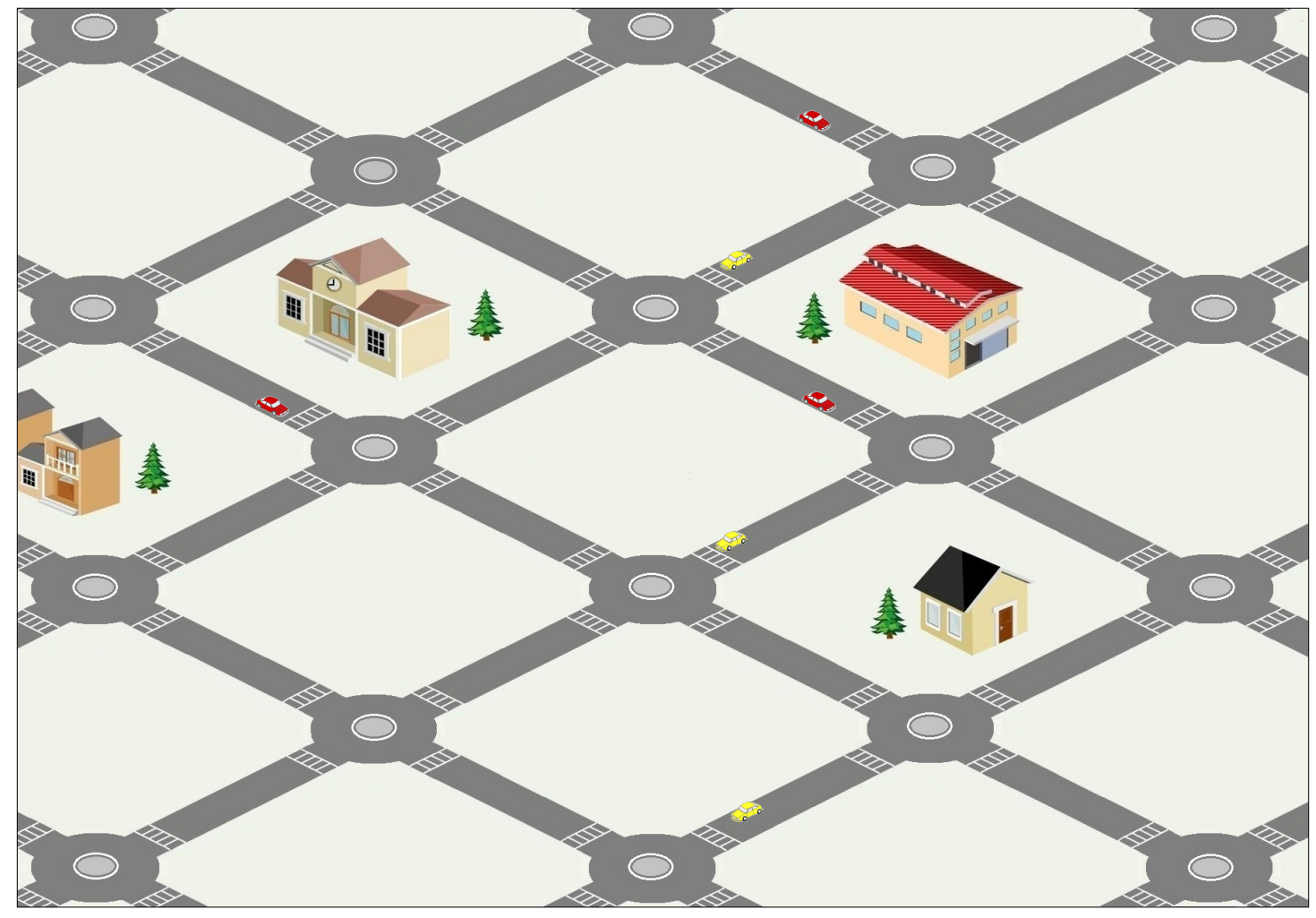

Fig. 1. The illustration of proposed structure of urban environment.

\section{Model description}

We consider a CA model described in the two-dimensional system with periodic boundary conditions. The underlying structure is a $L \times L$ cell grid, where $L$ is the system size. All streets are two-way, with one lane in each direction. The distance between each pair of roundabouts is configured with a parameter $K$ (see Fig. 2).

\subsection{Mobility model in urban streets}

In accordance to the NaSch model, we modeled our urban streets as a set of cells where each cell can either be empty or occupied by exactly one vehicle (see Fig. 2). The length of a single cell is set to $7.5 \mathrm{~m}$. The vehicles move within each route with periodic boundary conditions without changing lane. In each simulation setup, vehicles are either eastbound or westbound or southbound or northbound. The global density is defined by $\rho=N / L^{2}$. Here the city size $L$ is defined as $L=2 \sqrt{K * N_{r}}$, where $N_{r}$ is the number of roundabouts in the city.

At each discrete time step $t \longrightarrow t+1$, the system update is performed in parallel according to the following rules:

1. Acceleration outside roundabouts: $v_{n}(t+1)=\min \left(v_{n}(t)+1, v_{\max }\right)$

2. Deceleration: $v_{n}(t+1)=\min \left(v_{n}(t), d_{n}(t)\right)$

3. Randomization, braking: $v_{n}(t+1)=\min \left(v_{n}(t)-1,0\right)$ with probability $p$. 


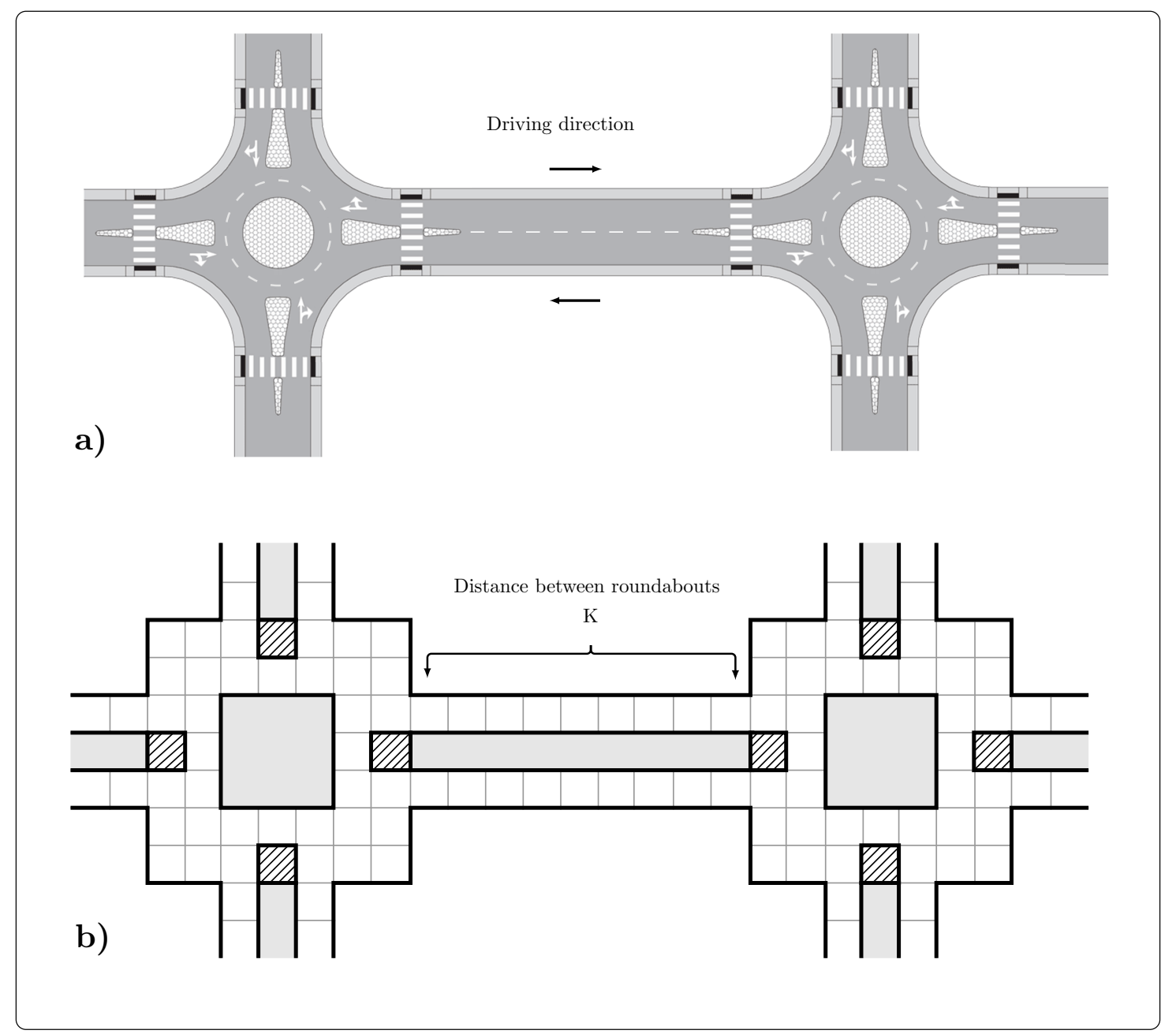

Fig. 2. Urban streets : (a) Realistic environment (b) CA model.

4. Car motion: each vehicle will move according to its new velocity.

where $v_{n}(t)$ denotes the velocity of the vehicle. $v_{\max }$ denotes the maximum velocity in urban streets. $d_{n}(t)$ denotes the gap (number of empty cells) between the vehicle $n$ and its immediate predecessor or between the vehicle $n$ and the last cell in the lane (cell that is right next to the roundabout).

\subsection{Mobility model and car accident in roundabout}

At each intersection between streets, we introduce a roundabout designed to allow drivers to change direction during their travel. Fig. 3 illustrates the type of roundabout considered in this paper. In addition, before entering the roundabout, each vehicle $n$ is assigned a turning probability $\left(p_{t u r n}(n)\right)$, which allows driver to choose his preferred driving direction (see Fig. 4). Note that in real world, the turning probability of different drivers may be distinct. For the sake of simplicity, we assume that of all drivers have the same turning probability $\left(p_{\text {turn }}(n)=\gamma\right)$. The turning behavior of vehicles at roundabouts is implemented via algorithm (1). 


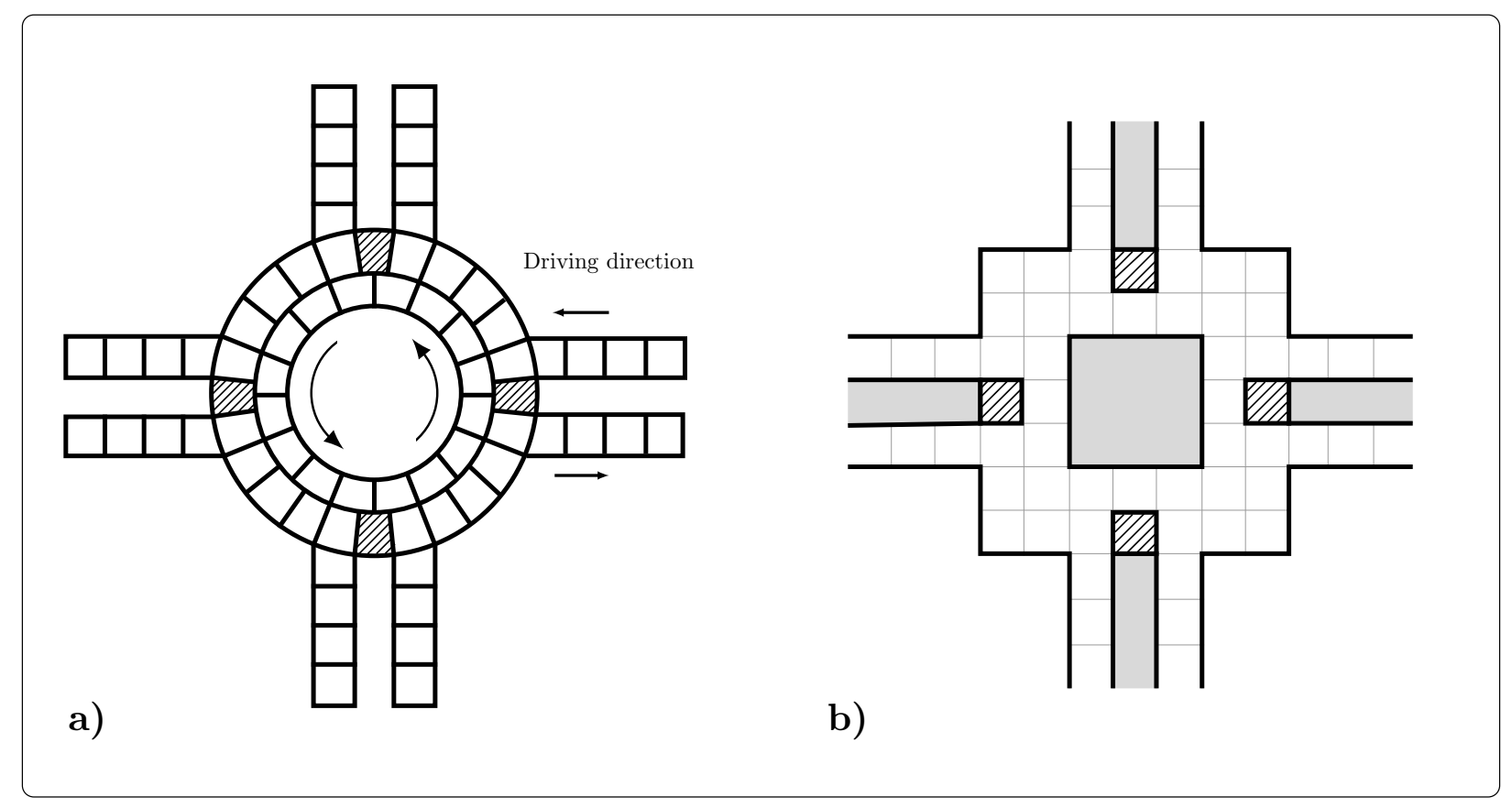

Fig. 3. Illustration of our proposed models of roundabout based on CA concept.

The majority of roundabouts were operated based on the offside priority rule, which implies that a vehicle $V_{A}$ approaching a roundabout (on the entry leg) is usually required to give way to the traffic already in the roundabout. Another priority rule contemplated here concerns another vehicle $V_{A}$ entering a roundabout and wishing to exit via the exit leg without using the circle of the roundabout. Let $V_{B}$ be a circulating vehicle in the roundabout that $V_{A}$ may encounter (see Fig. 5). Now, we define the set of gaps used to control the entering vehicle $V_{A}$ to roundabout based on offside priority rule. The gap $g a p_{A}^{1}(t)$ (resp. $\left.g a p_{B}^{1}(t)\right)$ represents the number of empty cells in front of the vehicle $V_{A}$ (resp. $V_{B}$ ) in its travel path at time $t$. For example in Fig. 5a, if the vehicle $V_{A}$ turn right then $g a p_{A}^{1}=g a p_{a}^{\prime}$ else $g a p_{A}^{1}=g a p_{a}$. Similarly, in Fig. 5b, if the vehicle $V_{B}$ turn left then $g a p_{B}^{1}=g a p_{d}$ else $g a p_{B}^{1}=g a p_{d}^{\prime}$. The gap $g a p_{A}^{2}(t)$ (resp. $\left.g a p_{B}^{2}(t)\right)$ represents the number of empty cells in front of the vehicle $V_{A}$ (resp. $V_{B}$ ) and the collision cell at time $t$. Remember that there are two different cells where a conflict may occur; the first is at the entry leg while the second is on the

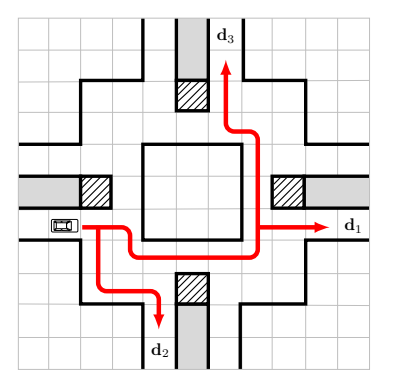

a)

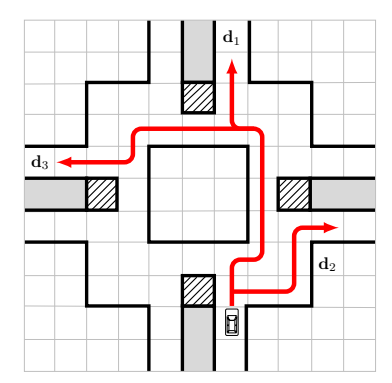

b)

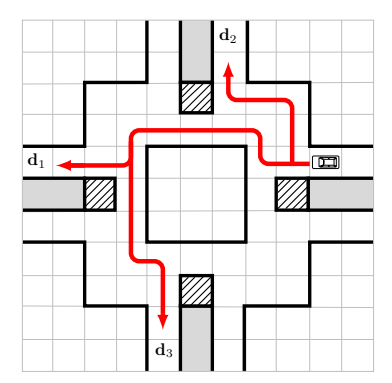

c)

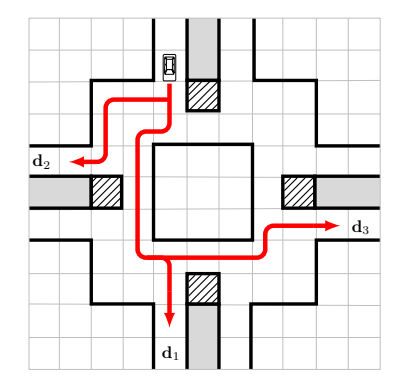

d)

Fig. 4. Illustration of allowed driving directions for drivers entering the roundabout. 


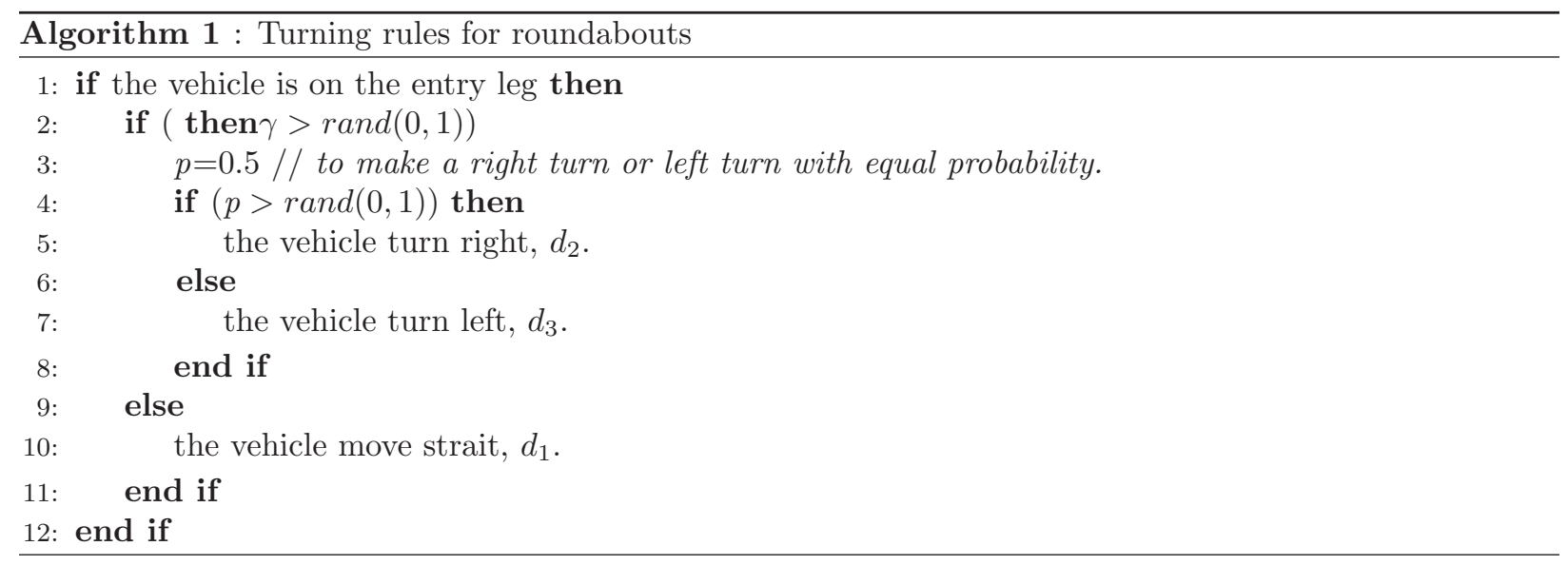

exit leg of the roundabout. In the situation where a conflict occurred at the entry leg (Fig. 5c), we have $g a p_{A}^{2}=g a p_{a}$ and $g a p_{B}^{2}=g a p_{b}$. However, at the exit leg (Fig. 5d), we have $g a p_{A}^{2}=g a p_{e}$ and $g a p_{B}^{2}=g a p_{f}$. The offside priority rule is implemented via the algorithm (2). So, if the speed of $V_{A}$ is large enough then the algorithm must be executed (line 1). The condition in line 2 means that when $V_{B}$ is far away from the cell of collision, $V_{A}$ enters safely to the roundabout. If not, $V_{A}$ will move to just behind the cell of collision (line 6). In the other hand, it is believed that roundabouts are very important for the limitation of traffic jams, the reduction of accidents and contribute to slow down speeding cars [18]. Nevertheless, roundabouts suffer from the occurrence of car accidents when entering vehicles violate the priority rules. The violation of safety conditions is mainly related to inability to accurately estimate the available gap, a lack of attention concerning the offside priority rule or the long reaction time $\tau$ of driver. The conditions of the occurrence of these accidents are defined as shown in algorithm 3.
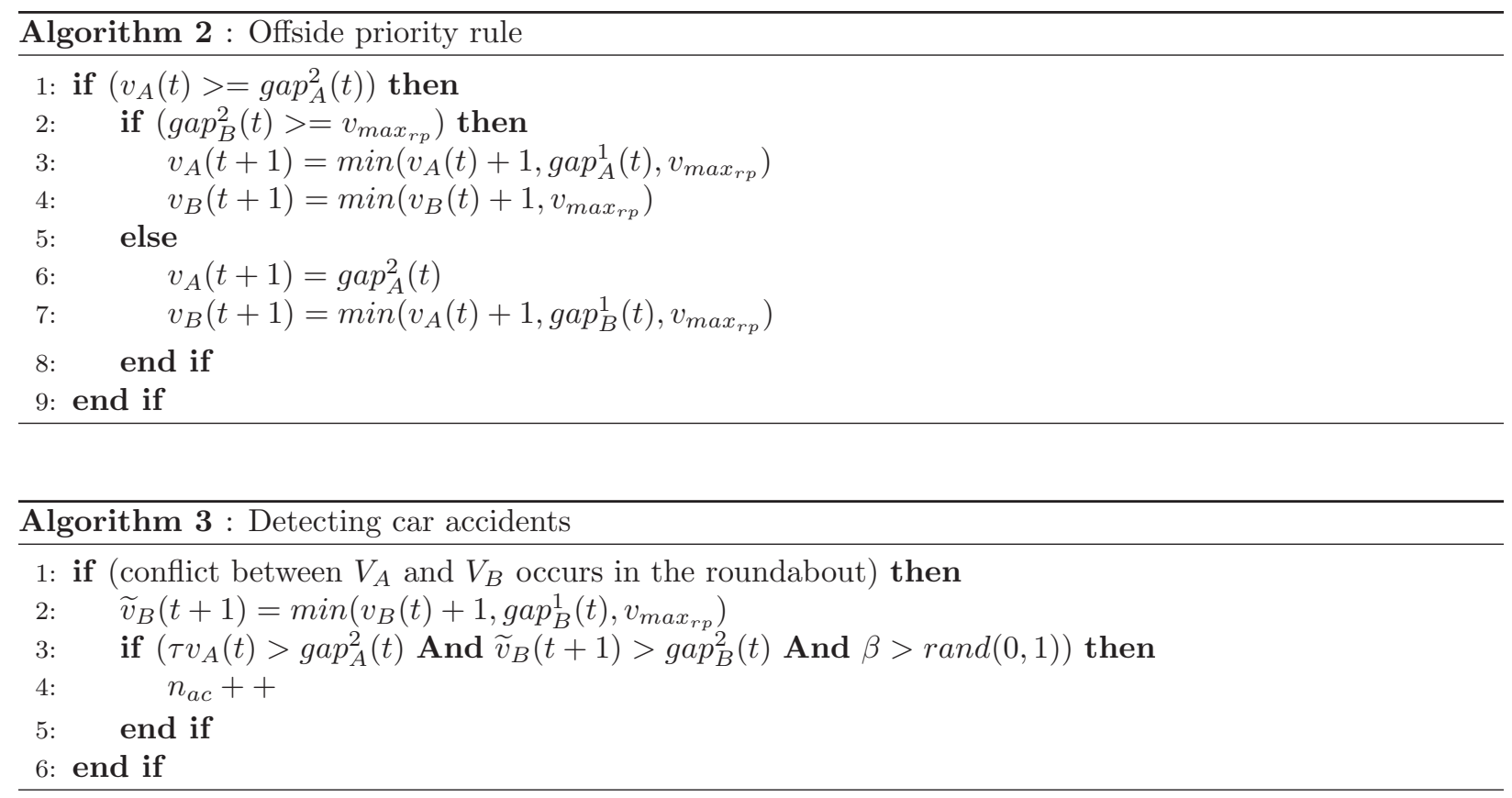


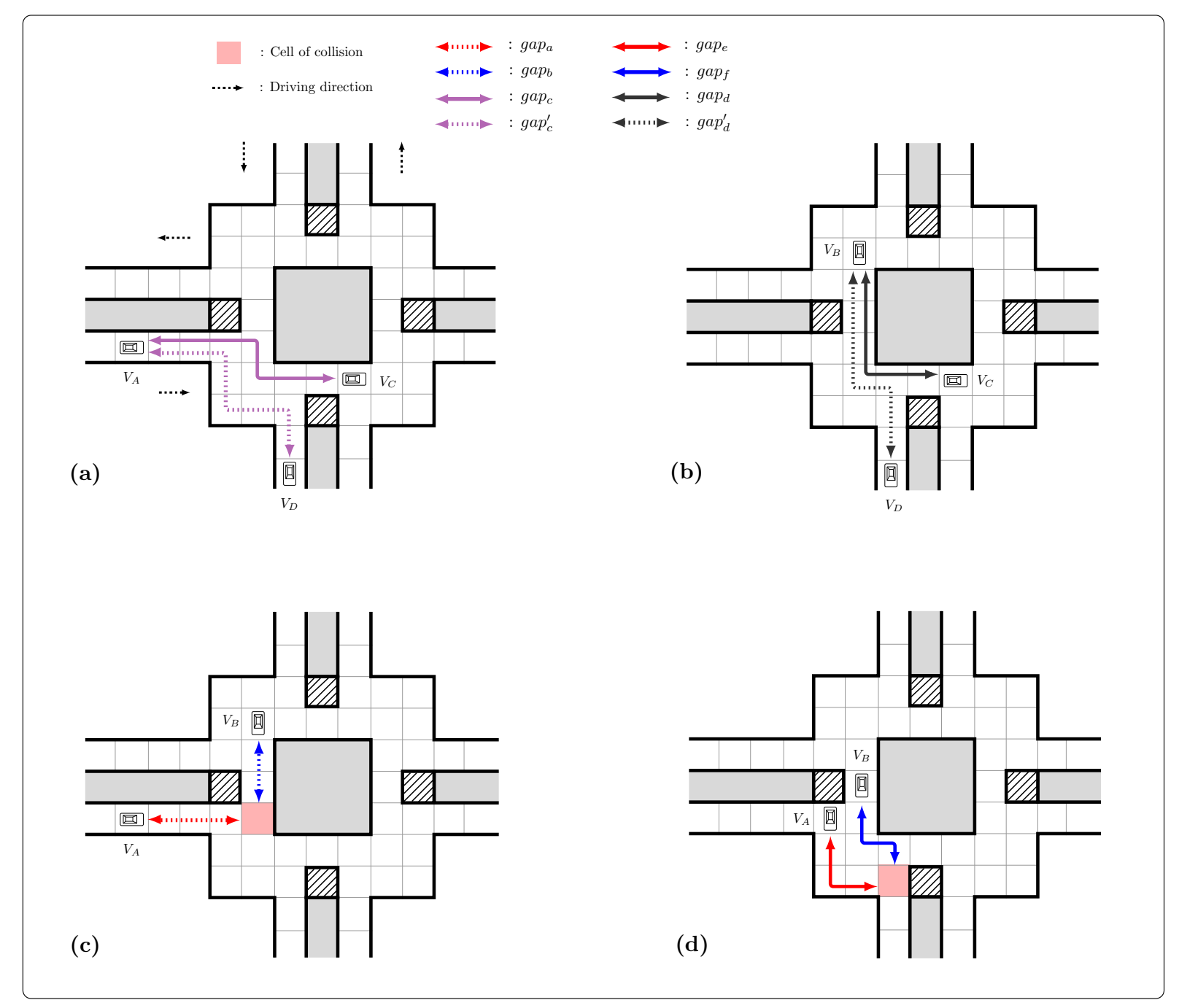

Fig. 5. Illustration of different gaps used to manage priority between entering vehicles and circulating vehicles and to determine car accidents at entry and exit legs of roundabout.

\subsection{Performance Metrics}

Model performance metrics are developed to assess our urban traffic system behavior. We monitor the impact of cars's density $\rho$ and turning probability $\gamma$ on traffic flow, accident probability and average waiting time. Similarly, we will investigate the impact of the urban geometric characteristics by varying the distance between roundabouts $K$, while keeping fixed the number of roundabouts $N_{r}$ in the urban city.

- Average velocity: we define the average velocity of vehicles as the sum velocities of all vehicles during the simulation time divided by time interval. The average velocity is then given as follows:

$$
\text { Average velocity }=\sum_{i=1}^{N} \frac{v_{i}(t)}{N \times T}
$$

where $\mathrm{T}$ is the time interval and $\mathrm{N}$ is the number of vehicles. 
- Traffic flow: we define the traffic flow as the car density $\rho$ multiplied to average velocity. The traffic flow in the city is then given as follows:

$$
\text { Traffic flow }=\rho \times \sum_{i=1}^{N} \frac{v_{i}(t)}{N \times T}
$$

- Accident probability: we define the accident probability as the total number of accidents $\left(\mathbf{n}_{a c}\right)$ committed by vehicles within the roundabout during the simulation time divided by the number of vehicles and the simulation time. The accident probability is then given as follows:

$$
\text { Accident probability }=\frac{n_{a c}}{N \times T_{s}}
$$

- Average waiting time: we define the waiting time as the time between the arrival time $\left(\mathbf{t}_{\text {arr }}^{i}\right)$ of a vehicle $i$ in the entry cell of a roundabout, and the moment of its departure $\left(\mathbf{t}_{d e p}^{i}\right)$ from this cell. Let $\mathbf{N}_{c}$ be the number of vehicles that have left the entry cells. The waiting time is then given as follows:

$$
\text { Average waiting time }=\frac{\sum_{i=1}^{N_{c}}\left(t_{d e p}^{i}-t_{a r r}^{i}\right)}{N_{c}}
$$

\section{Results and discussion}

In this section, we present the simulation results for our indicators of system performance in order to investigate the relationship between the states of our transportation system and the model parameters. The model parameters which have been considered in this work are given in Table 1. In this paper, we are interested to the effect of the turning rate and the city structure on the capacity of urban traffic. The impact of the city size is provided by varying the distance $K$ between roundabouts (Large cities correspond to high values of $K$ ).

First, we will analyze the different traffic states present in the transportation system. With increasing the density, we find that the traffic flow increases almost linearly and then begins to decrease beyond certain value of the density. This critical density $\rho_{c}$ represents a transition between free flow and congested state.

As Fig. 6 shows, this congested state is unstable and it depends greatly on the simulation time. A detailed study of this behavior leads to the discovery that congested states change over time to gridlock state when $\gamma \neq 0$. In this case, the model presents a state transition from free low to gridlock at a critical car density $\rho_{c}$. Fig. 7 shows a snapshot illustration of the two states present in the model when $\gamma=0.30$. In free-flow state (Fig. 7a) vehicles move freely while in gridlock state (Fig. 7b), vehicles pile up around some roundabouts, forming a single immobile cluster with zero mean velocity. However, on the other hand, for $\gamma=0$, we observe from Fig. 8a,c that the average velocity is stable with time and decreases when the density is diminished, thus showing the existence of the congested state. However, if $\gamma \neq 0$, the average velocity is stable with time for low values of the density; but as soon as this density exceeds $\rho_{c}$ the average velocity decreases with time and vanishes at $t=t_{\text {grid }}$; showing that the system has reached the gridlock state (Fig. $8 \mathrm{~b}, \mathrm{~d})$.

On the basis of our simulations, we can see that the main cause of the gridlock is the conflict of circulation between vehicles who circulate in a roundabout and those who want to leave. In such situations, 
Table 1. Mobility pattern parameters used in the simulation.

\begin{tabular}{lll}
\hline Parameter & Symbol & Value \\
\hline Roadmap size & $L \times L$ & - \\
Simulation time & $T_{s}$ & - \\
NaSch randomization probability & $p$ & 0 \\
Turning rate & $\gamma$ & - \\
Reaction time of driver & $\tau$ & $1 \mathrm{~s}$ \\
Car accident probability & $\beta$ & 0.3 \\
Maximum velocity for vehicles outside the roundabout & $v_{\max }$ & 3 \\
Maximum velocity for vehicles inside the roundabout & $v_{\max }$ & 2 \\
Number of roundabouts in the city & $N_{r}$ & 16 \\
Roundabout size & $R_{s}$ & 36 \\
Distance between roundabouts & $K$ & - \\
Time step increment & $d t$ & $1 \mathrm{~s}$ \\
\hline
\end{tabular}

no one can move because everyone is in someone else's way (??). More precisely, when several exits of a roundabout present simultaneously conflicts of circulation, a local deadlock emerges. This situation allows other circulating vehicles to slow down and stop because there is not a free space in front of exiting vehicles. This leads to the propagation of a traffic jam wave towards other roads linked to this roundabout, and thus causing a global gridlock.

In order to determine the critical density $\rho_{c}$, several simulations were carried out by examining the time evolution of the average velocity for different car densities. Fig. 9 shows the obtained results for different values of $\gamma$ and $K$. It is shown that the gridlock state can take longer time $\left(t_{\text {grid }}\right)$ to occur as the car density decreases, and $t_{\text {grid }}$ will become infinity when approaching to critical density. Our experimental simulations show that the model parameters have no obvious impact on the values of $\rho_{c}$. Moreover, since critical densities are found to be in the range of about 0.160 to 0.190 , Based on a detailed analysis of the
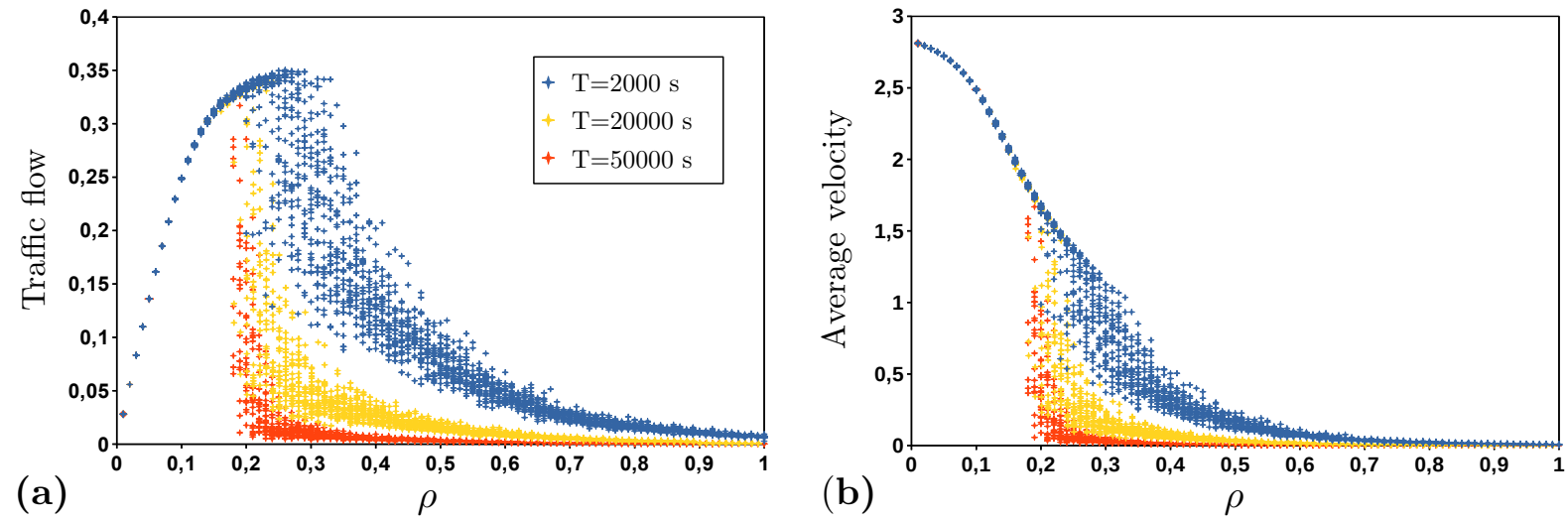

Fig. 6. Fundamental diagrams for different values of simulation time with parameters $K=40$ and $\gamma=0.3$. Each point represents one simulation run result and for each density we plot 30 points. (a) Traffic flow diagram (b) Mean velocity diagram. 
gridlock state, it appears that the gridlock occurs due to right-turning vehicles which may be trapped in loops when the car density exceeds a critical car $\rho_{c}$. This means that a loop formation can occur when the car density becomes sufficient to form a loop of vehicles. This loop is governed by the rate of right-turning vehicles especially when they are located in the head of road segments and waiting to access the roundabout, but the destination lanes are not empty which leads to a local gridlock. This local gridlock can propagate toward other sides of the city causing a global gridlock. As the appearance of gridlock is mainly related to an increase in the rate of right-turning vehicles in the road segments (e.g., $\gamma>0$ ) which lead to the formation the square loop.

However, when $\gamma=0$, the gridlock can occur, but with a much lower probability as compared with the occurrence of the square loop formed by the right-turning vehicles, and therefore the transition between free flow and gridlock can take much long time. As the right-turning vehicles are absent, the gridlock can occur only due to an intersection between fully saturated road segments that lead to make obstacles in front of exiting vehicles from the circulating ring of roundabout. We limited our results to $(\gamma=0.3)$ because

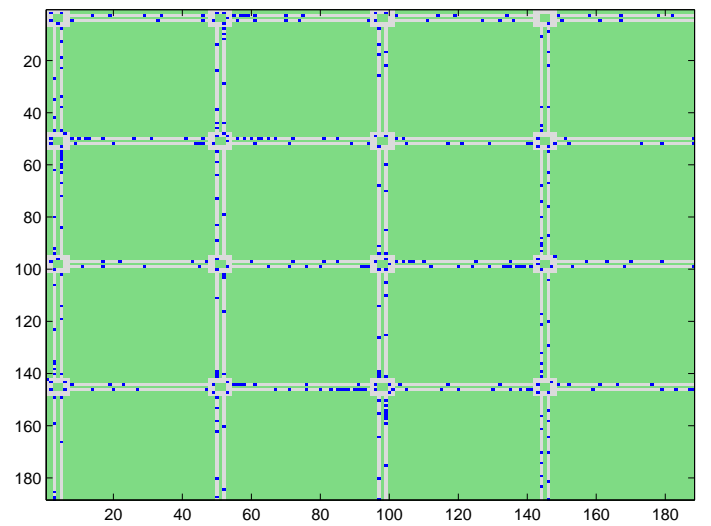

(a)

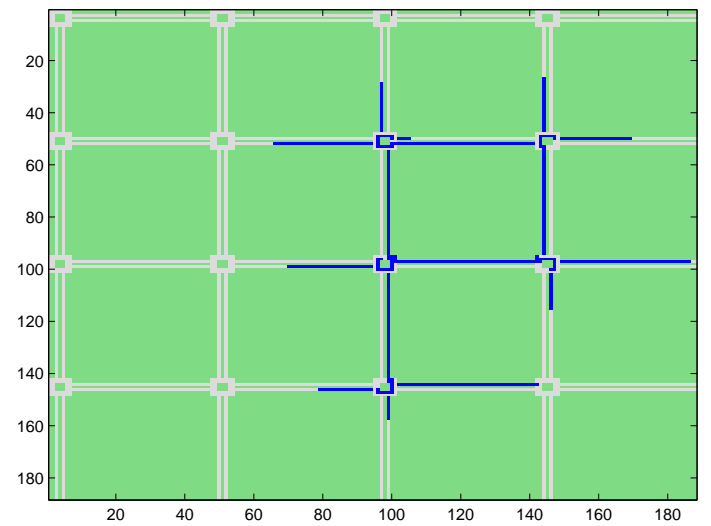

(b)

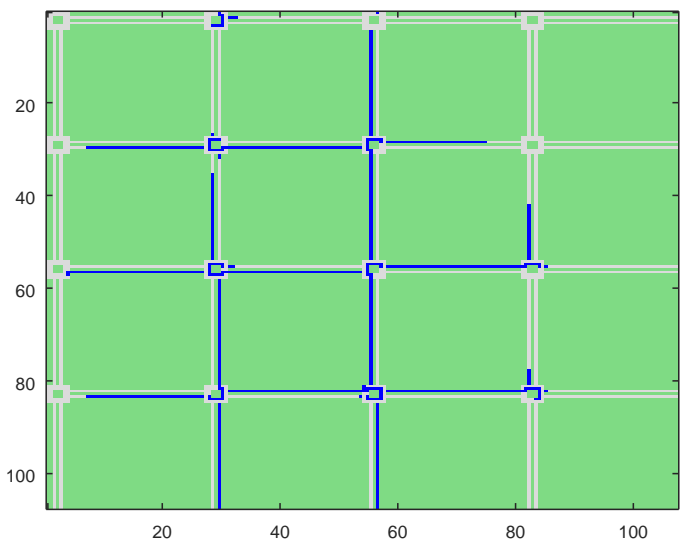

(c)

$$
\rho=0.3
$$

Fig. 7. Snapshot illustration of traffic states with parameters $K=40$ and $v_{\max }=3$. (a) $\rho=0.15$ and $\gamma=0.3$, (b) $\rho=0.2$ and $\gamma=0.3$, and (c) $\rho=0.3$ and $\gamma=0$. 
the detection of gridlock can take much long time (e.g., several days and months). But, when the density increases enough, it's passible to have a gridlock quickly as illustrated in (see Fig. 7c) for $(\rho=0.3)$. Indeed, we found that the critical car density can be given as follows:

$$
\rho_{c}=\frac{4 \times\left(K+R_{s}\right)}{32 \times K}
$$

Moreover, since critical densities are found to be in the range of about 0.160 to 0.190 , we limit our simulations and analysis up to the density 0.160 .

We begin our investigation by examining the impact of the turning rate on our traffic system. Fig. 10 shows the characteristics of traffic flow, average velocity, car accident and waiting time as a function of the density for different values of the turning probability $\gamma$. As plotted on Fig. 10a, the traffic flow versus the density reproduces the free flow state observed in real traffic flow, regardless of the value of $\gamma$. In this state, the traffic flow increases almost linearly with $\rho$ because almost all the vehicles move with their maximum speed $v=v_{\text {free }}$, due to the availability of large headways occurring in the traffic stream.

Furthermore, it is shown that $\gamma$ has no visible effect on the traffic flow and the average velocity for low car densities. As the car density increases $(\rho>0.1)$, turning movement starts to have an effect on the transportation system. It is shown that both traffic flow and average velocity increase with increasing $\gamma$, in accordance with the results of the model considered in [16]. This can be explained by the fact that
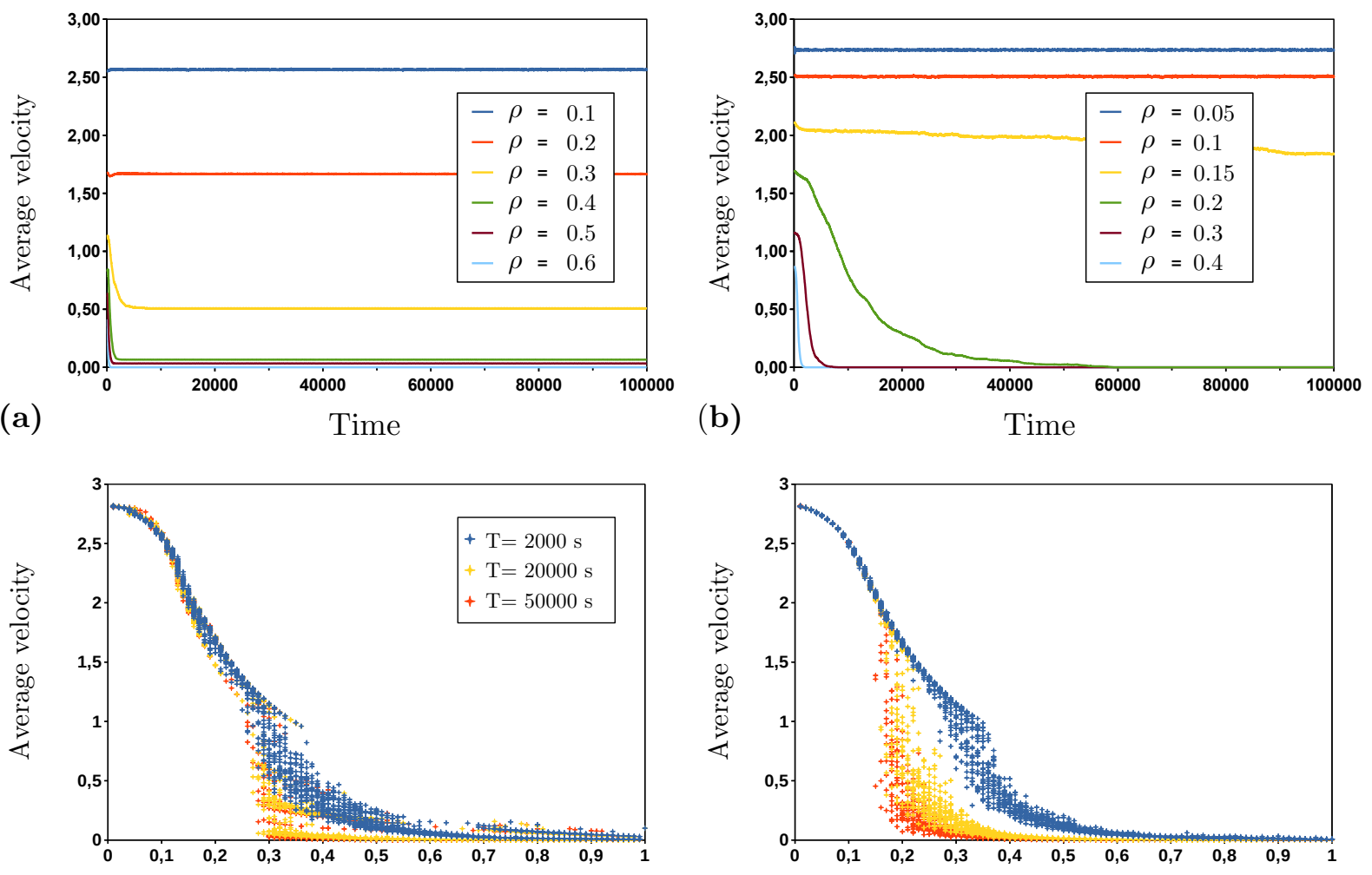

(c)

$\rho$

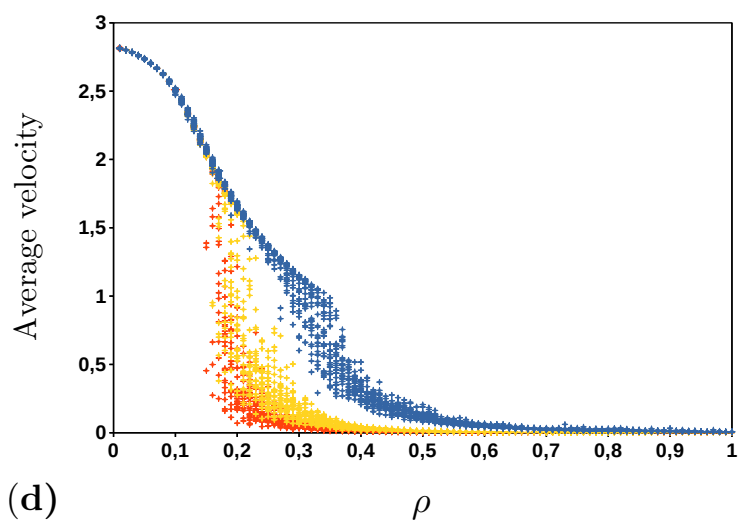

Fig. 8. Evolution of the average velocity for different car densities and simulation times with parameters $K=40$ and $\gamma=0.3$. (a) and $(\mathrm{c}): \gamma=0 ;(\mathrm{b})$ and $(\mathrm{d}): \gamma=0.05$. 
under high values of $\gamma$, the number of right turning movements is higher, and so several exit possibilities will be available for rotating vehicles. These exiting vehicles create opportunities for other entering vehicles, and therefore new gaps will be created inside roundabouts. Fig. 10c shows the car accident probability for different values of $\gamma$, as a function of car density. In this paper, we study the causes of car accidents at roundabouts, without taking into account their impacts on the traffic flow. This means that the car accident does not really happen, but only that we monitor the car accident probability without affecting the traffic flow. We observe that unlike accidents in highways, which usually do not occur until the cars density exceeds some critical value [14], accidents at roundabouts can exist even if the density is very low. Indeed, at low densities, vehicles access roundabouts at a maximal speed and the probability to collide with
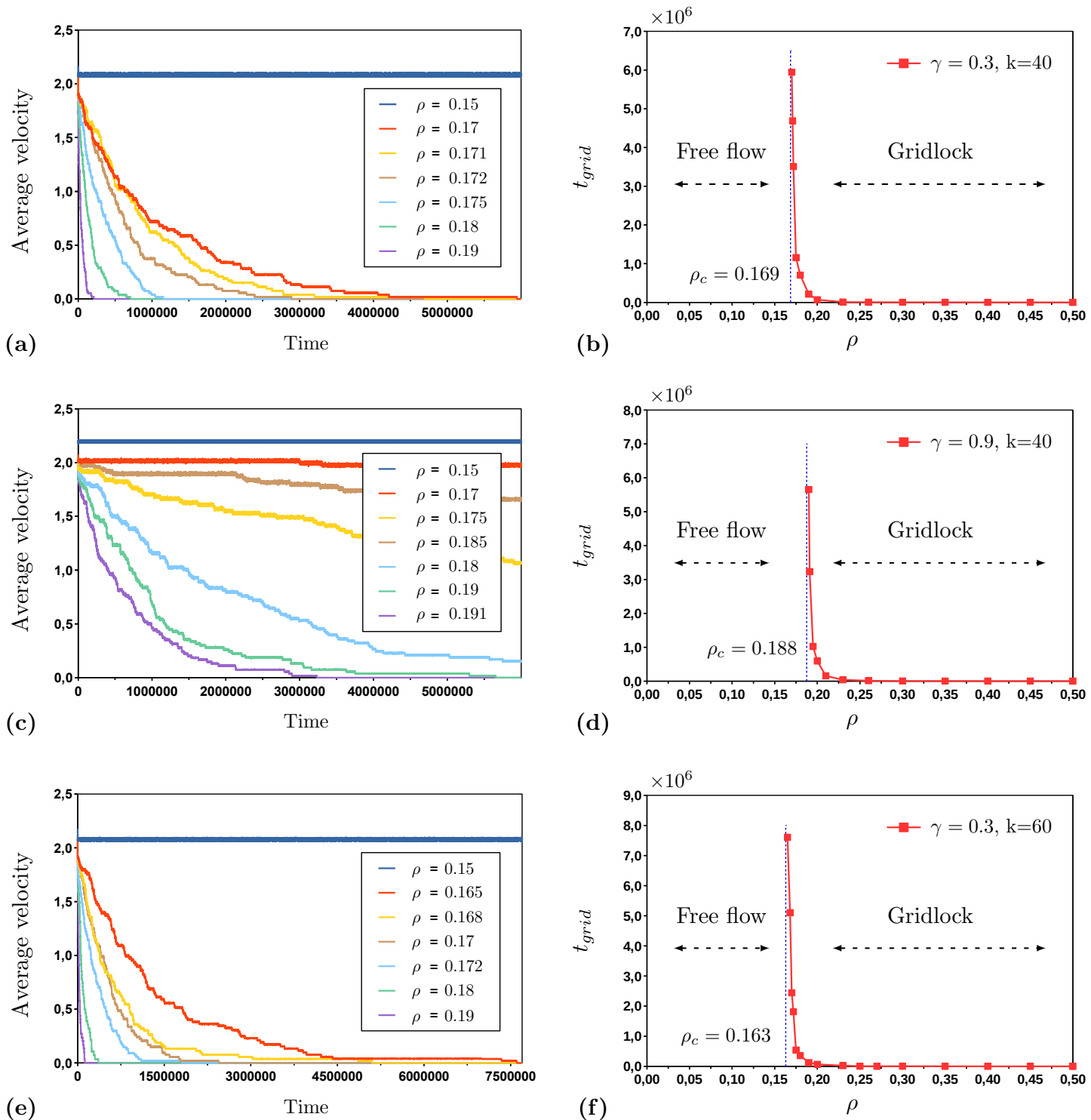

Fig. 9. Illustration of the transition between free flow and gridlock for different values of $\gamma$ and $K$. (a) and (b): $\gamma=0.3$ and $K=40$; (c) and (d): $\gamma=0.9$ and $K=40 ;(\mathrm{e})$ and (f): $\gamma=0.3$ and $K=60$ 

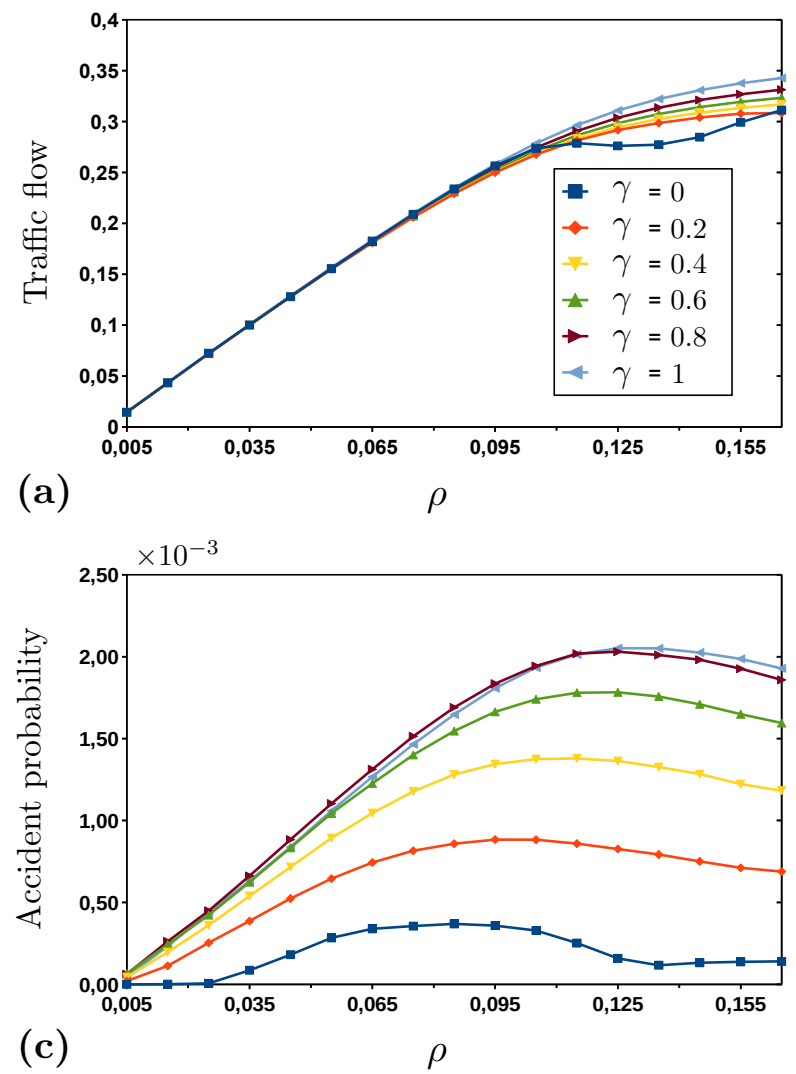
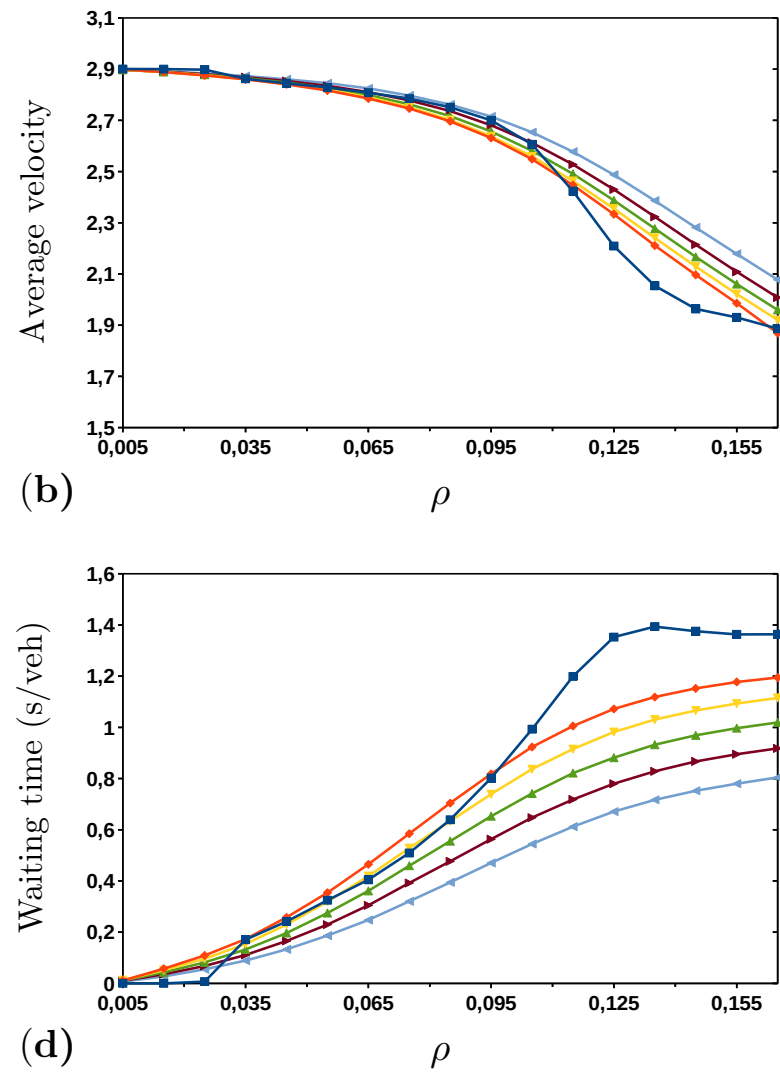

Fig. 10. Effect of increasing the turning probability $\gamma$ with $K=80$.

other circulating vehicles will be considerable. Moreover, with increasing the density, the car accident rate increases until it reaches a maximum, and then decreases with further density. It can be observed that, the higher the turning rate, the more frequent the accidents at roundabouts. Indeed, with higher values of $\gamma$, the traffic system creates more opportunities for entering vehicles and, as a result, these vehicles access the roundabouts with relatively high speeds, which increases the risk of collisions with rotating vehicles. On the other hand, for a given value $\gamma$, the waiting time increases as the density increases due to the excessive number of vehicles at roundabouts (see Fig. 10d). Moreover, at fixed density, the waiting time decreases with increasing $\gamma$. Once again, this is the result of the fluidity of traffic caused by the increase of $\gamma$.

In order to get a better understanding of the effect of urban network structure on the traffic dynamics, the plots of traffic flow, car accident probability, average velocity and waiting time against the density for varying values of $K$ are shown in Fig. 11. Note that, when we vary $K$, the number of roundabouts present in the urban structure remains fixed. Fig. 11a,b show that, as the distance between roundabouts $K$ decreases, the traffic flow and the average velocity decrease significantly. This can be explained by the fact that in the case of low values of $K$ (small cities), vehicles are able to reach the roundabouts quickly and therefore they are forced to either slow down or stop to avoid collisions with other circulating vehicles. This affects significantly the traffic flow and imposes a speed reduction of the vehicles every time they want to access the roundabouts (see Fig. 11b). From the obtained curves of Fig. 11c, it is clear that, at fixed car density, the high rate of car accidents corresponds to low values of $K$. As mentioned 

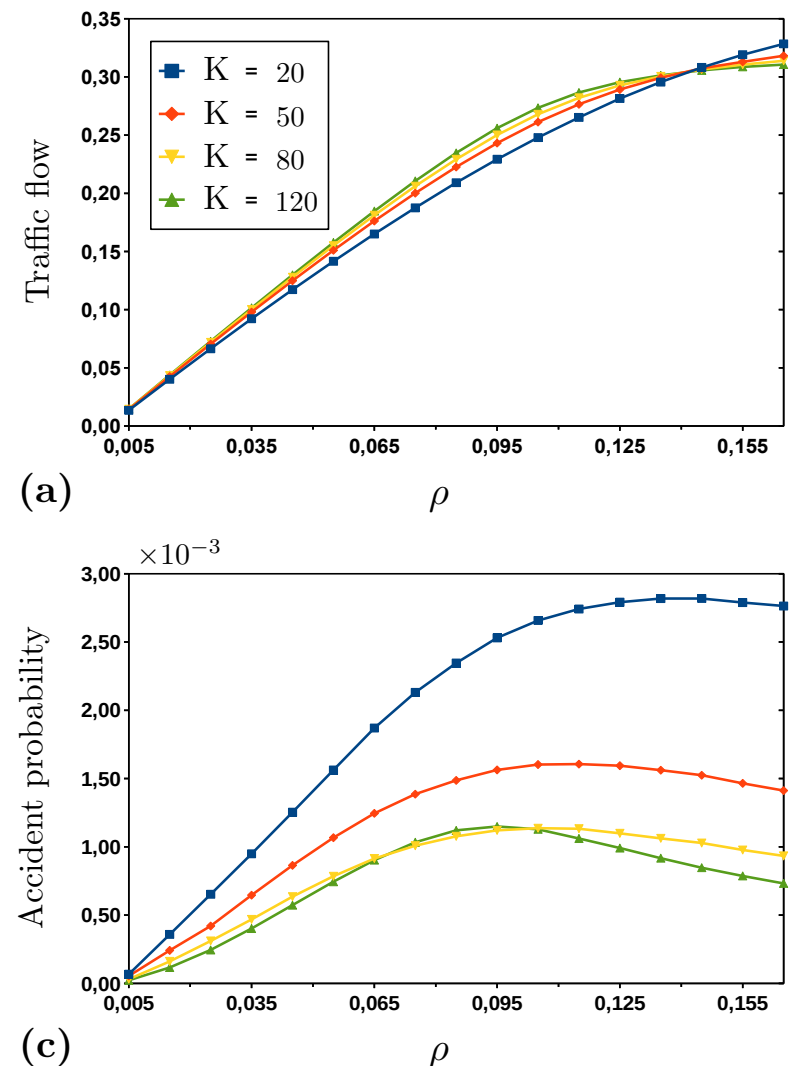
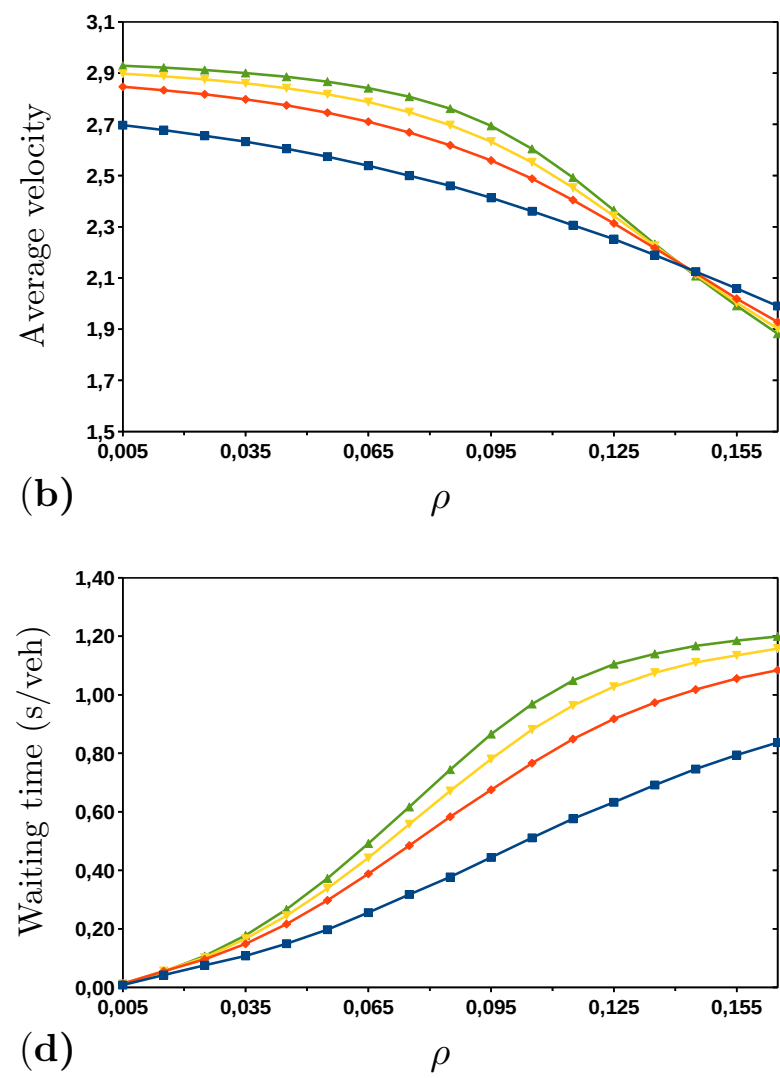

Fig. 11. Effect of increasing the distance between roundabouts $K$ with $\gamma=0.3$. The city size $L^{2}$ depends on the value of $K$ : $L^{2}=7200$ for $K=50, L^{2}=5760$ for $K=40, L^{2}=4320$ for $K=30$ and $L^{2}=2880$ for $K=20$.

before, the smaller the distance between two successive roundabouts, the more quickly vehicles reach the roundabouts. Therefore, during one simulation period, the frequency of violation of the safety conditions will become higher. Furthermore, for a given density, the waiting time increases with increasing $K$. In order to understand this phenomenon, it is necessary to remember that the number of roundabouts remains fixed when $K$ is increased. Moreover, for a fixed density, the increase in $K$ automatically leads to an increase in the number of vehicles $\left(\rho=N /\left(4 * N_{r} * K\right)\right)$. As a result, the traffic capacity of roundabouts is particularly limited if number of vehicles exceeds some limit. This will result in many stops in the entry legs and thus the average waiting time becomes more important.

\section{Conclusion}

Urban traffic with roundabouts has been studied based on a two dimensional CA model. Our results can be summarized as follows: 1. Urban traffic without turning behavior exhibits a transition from free flow to congested state as the density exceeds a critical density $\rho_{c}$. 2. The principal cause to the appearance of gridlock is the turning movement of vehicles. 3. Flow, accidents and waiting time are influenced by the turning rate as well as by the geometry of the urban city: - A high turning rate can lead to an increase in the urban traffic capacity, but also it can be a factor that increases the risk of accident. - Urban traffic is significantly better in large cities than in small ones. On the other hand, we have shown that $\rho_{c}$ varies 
very little when we vary the model parameters $\left(0.160<\rho_{c}<0.190\right)$. Then, it would be very interesting to implement intelligent strategies to increase $\rho_{c}$. This idea is under study and will be considered in a future work.

\section{References}

[1] K. Nagel, M. Schreckenberg, A cellular automaton model for freeway traffic, Journal de physique I 2 (12) (1992) $2221-2229$.

[2] D. Chowdhury, L. Santen, A. Schadschneider, Statistical physics of vehicular traffic and some related systems, Physics Reports 329 (4) (2000) 199-329.

[3] P.-g. Hou, H.-w. Yu, C. Yan, J.-y. Hong, An extended car-following model based on visual angle and backward looking effect, Chinese Journal of Physics 55 (5) (2017) 2092-2099.

[4] F. Járai-Szabó, Z. Néda, Earthquake model describes traffic jams caused by imperfect driving styles, Physica A: Statistical Mechanics and its Applications 391 (22) (2012) 5727-5738.

[5] M. Zamith, R. C. P. Leal-Toledo, E. Clua, E. M. Toledo, G. V. P. Magalhes, A new stochastic cellular automata model for traffic flow simulation with drivers behavior prediction, Journal of Computational Science 9 (2015) 51-56.

[6] B. Persaud, R. Retting, P. Garder, D. Lord, Safety effect of roundabout conversions in the united states: Empirical bayes observational before-after study, Transportation Research Record: Journal of the Transportation Research Board (1751) (2001) 1-8.

[7] S. Eisenman, J. Josselyn, G. List, B. Persaud, C. Lyon, B. Robinson, M. Blogg, E. Waltman, R. Troutbeck, Operational and safety performance of modern roundabouts and other intersection types, Project NYSDOT C-01 47.

[8] L. Rodegerdts, Roundabouts in the United States, vol. 572, Transportation Research Board, 2007.

[9] S. Mandavilli, A. McCartt, R. Retting, Crash patterns and potential engineering countermeasures at Maryland roundabouts, Traffic Injury Prevention 10 (1) (2009) 44-50.

[10] M. E. Fouladvand, Z. Sadjadi, M. R. Shaebani, Characteristics of vehicular traffic flow at a roundabout, Physical Review E 70 (4) (2004) 046132.

[11] R. Wang, H. J. Ruskin, Modelling traffic flow at multi-lane urban roundabout, International Journal of Modern Physics C 17 (05) (2006) 693-710.

[12] D.-w. Huang, Modeling gridlock at roundabout, Computer Physics Communications 189 (2015) $72-76$.

[13] N. Boccara, H. Fuks, Q. Zeng, Car accidents and number of stopped cars due to road blockage on a one-lane highway, Journal of Physics A: Mathematical and General 30 (10) (1997) 3329.

[14] N. Moussa, Car accidents in cellular automata models for one-lane traffic flow, Physical Review E 68 (3) (2003) 036127.

[15] H. Echab, N. Lakouari, H. Ez-Zahraouy, A. Benyoussef, Simulation study of traffic car accidents at a single lane roundabout, International Journal of Modern Physics C 27 (01) (2016) 1650009.

[16] J. A. Cuesta, F. C. Martínez, J. M. Molera, A. Sánchez, Phase transitions in two-dimensional traffic-flow models, Physical Review E 48 (6) (1993) R4175.

[17] L. G. Brunnet, S. Gonalves, Cellular automaton block model of traffic in a city, Physica A: Statistical Mechanics and its Applications 237 (1997) 59-66.

[18] A. Pratelli, Design of modern roundabouts in urban traffic systems, in: Urban Transport XII, eds. Brebbia and Dolezel, WIT Press, 83-93, 2006. 